\title{
Spatiotemporal Dynamics of Cerebral Vascular Permeability in Type 2 Diabetes-Related Cerebral Microangiopathy
}

\section{Ying-Chen Chen}

National Cheng Kung University Hospital

\section{Bing-Ze Lu}

National Cheng Kung University

\section{Yu-Chen Shu}

National Cheng Kung University

Yuan-Ting Sun ( $\nabla$ ytsun@mail.ncku.edu.tw)

National Cheng Kung University College of Medicine https://orcid.org/0000-0003-2899-1136

Original investigation

Keywords: diabetes mellitus, blood-brain barrier, permeability, cerebral small vessel disease

Posted Date: October 21st, 2021

DOl: https://doi.org/10.21203/rs.3.rs-970765/v1

License: (a) (i) This work is licensed under a Creative Commons Attribution 4.0 International License.

Read Full License

Version of Record: A version of this preprint was published at Frontiers in Endocrinology on January 11th, 2022. See the published version at https://doi.org/10.3389/fendo.2021.805637. 


\section{Abstract \\ Objective}

Diabetes-related cerebral microangiopathy can manifest as cerebral small vessel disease (CSVD) and exhibit cognitive decline. To find the early change of function in advance, this study examined the spatiotemporal dynamics of cerebral permeability $\left(K_{\text {trans }}\right)$ in the progression of diabetes-related CSVD.

\section{Methods}

Cerebral vascular permeability was crossectional measured in diabetic patients with or without CSVD, and non-diabetic patients with or without CSVD by using dynamic contrast-enhanced MRI (DCE-MRI).

\section{Results}

In all diabetic patients, the $K_{\text {trans }}$ of white matter (WM) was increased. However, the $K_{\text {trans }}$ of gray matter (GM) was only increased in those with CSVD. This suggested the earlier involvement of WM than GM and indicated the development of diabetes-related cerebral microangiopathy was prior to it could be visualized as features of CSVD on MRI. To broaden the application of cerebral permeability and overcome the limitations of DCE-MRI, the commonly available CSVD items of MRI were used to indicate the increase in $K_{\text {trans }}$. Among all CSVD items, the presence of microbleeds was most correlated with the increased permeability in all patients. In contrast to non-diabetic patients, increased $K_{\text {trans }}$ in diabetes was more associated with moderate WM hyperintensity but less with the presence of lacunae or multiple perivascular spaces. The differential correlation suggested distinct mechanisms underlying diabetesrelated CSVD and other CSVDs.

\section{Conclusions}

This study highlights the early development of cerebral microangiopathy in diabetes and broadens the applicability of cerebral permeability. The results may increase the proactivity of clinicians in recognizing the subsequent neurological comorbidities.

\section{Introduction}

Cerebral small vessel disease (CSVD) represents a heterogeneous group of disorders. The hypothetical aetiologies include microatheroma, endothelial dysfunction, inflammation, and altered microvascular blood-brain barrier (BBB) integrity ${ }^{1}$. Diabetes is highly associated with CSVD ${ }^{2-4}$ that can manifest as symptomatic or silent lacunar infarcts and cognitive decline. Diabetes-related CSVD can be diabetic microangiopathy involving the brain. Given the increasing global prevalence of diabetes, CSVD and the 
accompanying neurological disability are considerable challenges for healthcare systems ${ }^{5}$ and thus are becoming increasingly crucial ${ }^{4-7}$.

Vascular permeability was recently reported as a critical mechanism underlying dementia ${ }^{8}$. Diabetes triggers various vascular pathologies, including increased vascular permeability that may contribute to cognitive decline and other morbidities such as ischemic stroke ${ }^{9,10}$. Therefore, the increase in BBB permeability in patients with diabetes might be an early tissue-level dysfunction associated with subsequent cognitive decline.

This study hypothesized that the increase in vascular permeability would present early in patients with diabetes, even before the development of CSVD features on MRI. This temporal evolution may be unique to diabetes-related CSVD that represents cerebral microangiopathy similar to other target organs in diabetes. To test the hypothesis, we performed dynamic contrast-enhanced MRI (DCE-MRI) to examine the spatiotemporal dynamics of cerebral vascular permeability ${ }^{11}$ in diabetes patients with or without CSVD. Then we correlated the CSVD items of conventional MRI with cerebral permeability and compared the findings with CSVD patients without diabetes to identify the unique features of the diabetes-specific cerebral microangiopathy in a heterogeneous CSVD group.

\section{Materials And Methods}

\section{Study design}

This study was carried out at the National Cheng Kung University Hospital (Tainan, Taiwan) and Kaohsiung Municipal United Hospital (Kaohsiung, Taiwan). The local Institutional Review Board approved the protocol (Approval No. A-BR-106-081). Four groups of subjects, diabetic patients without CSVD, diabetic patients with CSVD, patients with CSVD but without diabetes, and controls (individuals without diabetes, without CSVD), were recruited from the neurology outpatient clinic in National Cheng Kung University Hospital. Demographic data, permeability, CSVD scores on MRI between the four groups were analyzed cross-sectionally.

\section{Participants}

In recruiting subjects, the diagnosis of type 2 diabetes, followed by the American Diabetes Association ${ }^{12-}$ 14 , should exist on medical records for at least five years. The stages of chronic kidney disease (CKD) were followed the guidance from National Kidney Foundation ${ }^{15}$. Hypertension, if it exists, should be well controlled $^{16}$. The presence of CSVD was defined as a total CSVD score of $>1$ on MRI ${ }^{17}$. Patients with intracranial tumor, cerebral venous thrombosis, hemorrhagic stroke, immune-mediated neuroinflammatory disease, hydrocephalus, $>50 \%$ stenosis in any large intracranial vessel, hereditary neurodegenerative disorder, advanced CKD (> stage III), liver cirrhosis, history of posterior reversible encephalopathy syndrome, and acute infection were ineligible for this study. Demographic data, dementia, hypertension, hyperlipidemia, levels of $\mathrm{HbA1c}$, total cholesterol, low-density lipoprotein 
cholesterol, and creatinine were collected. Patients with a Mini-Mental Status Examination score of $\leq 26$ or a Clinical Dementia Rating score of $\geq 0.5$ were considered to have dementia.

\section{Imaging protocol}

\section{Structural imaging}

All participants underwent conventional structural imaging and then DCE-MRI on an MRI system (Ingenia 3.0 T, Philips Healthcare, the Netherlands). Conventional structural sequences contained axial T1weighted images, axial T2-weighted fat-saturation images, and axial T2-weighted fluid-attenuated inversion recovery (FLAIR) images, as well as susceptibility-weighted images to evaluate microbleeding.

\section{DCE-MRI}

To examine vascular permeability on MRI, two reference series using axial T1-weighted fast-field echo (T1-FFE) with flip angles of $5^{\circ}$ and $15^{\circ}$ were initially performed. Subsequently, DCE-MRI of the whole brain was performed using axial T1-FFE with a flip angle of $8^{\circ}$ (repetition time/echo time, 2.8/1.32 ms; slice width, $3 \mathrm{~mm}$; no slice gap; field of view, $230 \times 202 \times 108 \mathrm{~mm}^{3}$; matrix, $\left.116 \times 115\right)$. An intravenous bolus injection of a gadolinium-based contrast agent, gadoterate meglumine (Gd-DOTA), was administered after the first imaging of this series at a dose of $0.1 \mathrm{mmol} / \mathrm{kg}$. The DCE-MRI sequence was repeated 63 times over a 25-min acquisition period. The details of imaging intervals are as follows: an imaging interval of $4 \mathrm{~s}$ during a postcontrast injection of 0-1 min, an imaging interval of $6 \mathrm{~s}$ during a postcontrast injection of 1-3 min, an imaging interval of $8 \mathrm{~s}$ during a postcontrast injection of 3-4 min, and an imaging interval of $1 \mathrm{~min}$ during a postcontrast injection of 4-25 min (Fig. 1A).

\section{Image analysis}

To overcome the subtle inhomogeneity between T1 images, we used the intensity of the fat tissue near the constant brightness of each image as a reference for the following calibration. The averaged intensity of the three regions of fat around the eyes was adjusted to a value of 1 in each image. Five regions of interest (ROIs) were chosen: frontal WM, parietal WM, caudate head (CH), putamen, and thalamus. The frontal and parietal WM measurements were obtained using an axial view image at the level above the $\mathrm{CH}$. The centers of the ROls were laterally next to the frontal and parietal horns of the lateral ventricles (Fig. 1B). The $\mathrm{CH}$, putamen, and thalamus measurements were obtained using an axial view image at the central thalamus and Sylvian fissure (Fig. 1C). To avoid bias caused by the inhomogeneity of the selected $\mathrm{ROIs}$, the center and radius of each ROI were randomly oscillated within 3 pixels to obtain six ROls close to the chosen ROI (Fig. 1D) initially. The intensities of the six corresponding oscillated regions were averaged to represent the selected ROI initially.

Determination of BBB permeability ( $\left.K_{\text {trans }}\right)$ 
A Patlak graphical analysis technique was adopted to determine pixel-wise BBB permeability $\left(K_{\text {trans }}\right)$ in an $\mathrm{ROI}$ under the assumption of the irreversible uptake pharmacokinetics of Gd-DOTA ${ }^{18}$. In a compartment model, the relative concentration $C(\mid \operatorname{varvec} r, t)$ was modeled in terms of the contrast of the initial intensity as follows:

$$
C(\boldsymbol{r}, t)=-\frac{K}{T_{e}} \ln \left(\frac{S(\boldsymbol{r}, t)}{S(\boldsymbol{r}, 0)}\right)
$$

where $T_{e}$ is the echo time, $\mathrm{K}$ is the concentration constant, and $S(\mid \operatorname{varvec} r, t)$ is the intensity of the pixel location Ivarvec $r$ at time $t$ after Gd-DOTA injection. The concentration $C_{i}(\mid \operatorname{varvec} r, t)$ in the ROI was estimated using the following equation:

$$
C_{i}(\boldsymbol{r}, t)=K_{\text {trans }}(\boldsymbol{r}) \int_{0}^{t} C_{p}(\tau) d \tau+V(\boldsymbol{r}) \cdot C_{p}(t)
$$

where $V(\backslash$ varvec $r)$ is the distribution of Gd-DOTA in the vessels, $C_{p}(t)$ is the concentration of Gd-DOTA in the plasma, and $K_{\text {trans }}(\backslash$ varvecr $r)$ is the rate at which Gd-DOTA enters the brain parenchyma, indicating permeability. We obtained the plasma Gd-DOTA intensity by marking the internal carotid artery as the region of plasma (ROP; Fig. 1E). In addition, we perturbed the selected ROP to reduce the inhomogeneity between pixel values. The averaged relative concentration of ROP was calculated using the following equation:

$$
C_{p}(t)=\iint_{r \in \mathrm{ROP}} C(\boldsymbol{r}, t) d A / \iint_{r \in \mathrm{ROP}} d A .
$$

\section{Noise elimination}

To minimize noise produced by imaging procedures, a noise elimination method that involved plotting the histogram of $K_{\text {trans }}$ in an ROI was applied ${ }^{19}$. After all the $K_{\text {trans }}$ values of each pixel were computed, normalization preprocessing was performed to obtain the pixel-wise proportion of each $K_{\text {trans }}$. The cumulative sum of all $K_{\text {trans }}$ values was set as 1 . Subsequently, all $K_{\text {trans }}$ values were plotted pixel-wise on a histogram, with the proportion of the pixel in an ROI presented on the y axis (Fig. 1F). Because $K_{\text {trans }}$ in the model refers to the rate of entry into an irreversible compartment, a negative value representing an opposite direction of the movement of Gd-DOTA from the parenchyma to the vessel was considered noise (red bars in Fig. 1F). In addition, the noise was supposed to be present on the positive part of the histogram with a mirror distribution of the negative part. After subtracting the estimated noise (red bars, Fig. $1 F$ ) from the positive $K_{\text {trans }}$ histogram (blue bars, Fig. 1F), the remaining cumulative sum of the bars was identified as the approximate $K_{\text {trans }}$ in an ROI (Fig. 1G).

\section{CSVD scoring}


The presence of CSVD was determined by viewing structural MRI images and calculating the total CSVD score through the summation of four parameters: lacunes, microbleeds, perivascular space (PVS), and grading of periventricular leukoaraiosis, namely WM hyperintensity $(\mathrm{WMH}){ }^{17}$. The WMH was graded from 0 to 3 using the Fazekas scale. Patients were considered to have CSVD if their CSVD score was $>1$.

\section{Software and Statistical Analysis}

The total $K_{\text {trans }}$ represented an average from $K_{\text {trans }}$ of WM and $K_{\text {trans }}$ of GM. $K_{\text {trans }}$ of WM was an average from $K_{\text {trans }}$ of frontal and parietal WM. $K_{\text {trans }}$ of GM was an average from $K_{\text {trans }}$ of $\mathrm{CH}$, putamen and thalamus. The imaging processing and scientific computing of $K_{\text {trans }}$ were performed using MATLAB (2019b, The MathWorks, Natick, Massachusetts, USA) and the Python programming language (version 3.7). Statistical analyses were performed using Prism (version 6; GraphPad Software, La Jolla, CA, USA) and the $\mathrm{R}$ programming language (version 4.0.4). Uunpaired Student's $t$-test, Mann-Whitney U test, Fisher's exact test, one-way analysis of variance (ANOVA), or Kruskal-Wallis test were used according to data type and group number. Normality tests were conducted for continuous data before comparisons. To test the correlation, Kendall's tau was used for ordinal data, and a permutation-based linear model with 10,000 random shufflings was used for continuous numerical data. Significance was set at $P<0.05$.

\section{Results}

Thirty-one patients with diabetes and 14 individuals without diabetes were enrolled. Seven were excluded because of numerous motion artifacts on their MRI. Finally, 25 patients with diabetes (11 women, 49-88 years old) and 12 individuals without diabetes (8 women, 21-81 years old) were included in the analysis. The demographic data of the excluded subjects were not different from the study subjects. Patients with diabetes were further divided into two groups based on the MRI-based CSVD score: diabetes without CSVD ( $n=12,7$ female, 49-78 years old) and diabetes with CSVD ( $n=13,4$ female, 63-88 years old) (Table 1). The distribution of sex and the prevalence of hypertension did not differ among the three groups $(P=0.25$ and 0.18 , respectively, Fisher's exact test). Subjects of diabetes with CSVD were older ( $P$ $=0.004$, one-way ANOVA with the multiple comparisons test), had a higher proportion of dementia $(P=$ 0.034 , Fisher's exact test), and a more advanced stage of CKD ( $P=0.036$, Kruskal-Wallis test). Among patients with diabetes, the HbA1c level was equal $(P=0.22$, Student's t-test) between the CSVD and nonCSVD groups. The scores of individual CSVD items and the total CSVD score did not differ between the control and diabetes without CSVD groups (total score, $\mathrm{P}=0.301$; lacune, $\mathrm{P}=0.9 ; \mathrm{PVS}, \mathrm{P}=0.39 ; \mathrm{WMH}, \mathrm{P}$ = 0.62; microbleeds, $\mathrm{P}=0.195 ;$ Mann-Whitney U test). 
Table 1

Demographic data of patients with and without diabetes

\begin{tabular}{|c|c|c|c|c|c|}
\hline & \multirow{2}{*}{$\begin{array}{l}\text { Total } \\
(\mathbf{N}=37)\end{array}$} & \multicolumn{2}{|c|}{ diabetes $(\mathrm{N}=25)$} & \multirow{2}{*}{$\begin{array}{l}\text { Non-diabetes } \\
(\mathrm{N}=12)\end{array}$} & \multirow[t]{2}{*}{ p-value } \\
\hline & & $\begin{array}{l}\text { CSVD } \\
(\mathrm{N}=13)\end{array}$ & $\begin{array}{l}\text { No CSVD } \\
(\mathrm{N}=12)\end{array}$ & & \\
\hline Gender (Female,\%) & $19(51.4)$ & $4(30.7)$ & $7(58.3)$ & $8(66.7)$ & 0.257 \\
\hline Age (mean \pm SD) & $\begin{array}{l}64.83 \pm \\
15.26\end{array}$ & $\begin{array}{l}74.61 \pm \\
8.75\end{array}$ & $63.25 \pm 10.4$ & $55.83 \pm 19.32$ & $0.004^{\star *}$ \\
\hline Hypertension ( $\mathrm{N}, \%)$ & $20(54.1)$ & $8(61.5)$ & $8(66.7)$ & $5(33.3)$ & 0.313 \\
\hline Dementia $(\mathrm{N}, \%)$ & $16(43.2)$ & $10(76.9)$ & $6(50)$ & $3(25)$ & $0.034^{*}$ \\
\hline $\begin{array}{l}\text { CKD stage (median, } \\
\text { [IQR]) }\end{array}$ & $1[1,2]$ & $2[1.5,3]$ & $1[1,2]$ & $1[1,1]$ & $0.036^{*}$ \\
\hline $\begin{array}{l}\text { DM, diabetes mellitı } \\
\text { deviation; * } \mathrm{P}<0.05\end{array}$ & $\begin{array}{l}\text { D, cereb } \\
0.01\end{array}$ & all vess & ase; CKD, ch & kidney diseas & andard \\
\hline
\end{tabular}

Temporal dynamics: diabetes showed an impact on the increase in $\mathrm{K}_{\text {trans }}$ in addition to the effect of aging, particularly in diabetes with CSVD group

In all subjects, the total $K_{\text {trans }} K_{\text {trans }}$ of WM, and $K_{\text {trans }}$ of GM all increased with age (Fig. 2D-G), approximately following the equation of total $K_{\text {trans }}=(0.2517$ * age -4.254$) / 1000\left(R^{2}=0.3638, P=\right.$ $0.0379)$ in control group and total $K_{\text {trans }}=[0.1768 *$ age +12.73$] / 1000$ in diabetes group. Before the adjustment for age, the diabetes group had increased total $K_{\text {trans }} K_{\text {trans }}$ of WM, and $K_{\text {trans }}$ of GM (P = $0.0258,0.0199$, and 0.0319 , respectively, Student's $t$-test, Fig. 2A-C). In order to adjust the effect of aging, a permutation-based linear model with 10,000 random shufflings of patients and healthy controls was performed to test the correlation. A significant effect of diabetes on the $K_{\text {trans }}$ of WM $(p=0.048$, Fig. $2 F)$ but not on total $K_{\text {trans }}$ and $K_{\text {trans }}$ of GM was found ( $\mathrm{p}=0.128, \mathrm{p}=0.132$, respectively, Fig. 2E-G) between diabetes and control group. 
Table 2

Statistical power of various combinations of CSVD items in determining $K_{\text {trans }}$ among patients with diabetes

\begin{tabular}{|c|c|c|}
\hline Criteria & Sensitive region & p-value \\
\hline \multirow[t]{3}{*}{$\mathrm{MB} \geq 1$} & $\mathrm{CH}$ & $0.044^{\star}$ \\
\hline & putamen & $0.047^{*}$ \\
\hline & parietal WM & $0.04^{*}$ \\
\hline \multirow[t]{2}{*}{$\mathrm{MB} \geq 1$ and $\mathrm{WMH} \geq 1$} & $\mathrm{CH}$ & $0.044^{\star}$ \\
\hline & parietal WM & $0.04^{*}$ \\
\hline \multirow[t]{3}{*}{$\mathrm{MB} \geq 1$ and $P V S \geq 2$} & $\mathrm{CH}$ & $0.044^{*}$ \\
\hline & putamen & $0.047^{*}$ \\
\hline & parietal WM & $0.04^{*}$ \\
\hline \multirow[t]{3}{*}{$\mathrm{MB} \geq 1$ and $\mathrm{PVS} \geq 3$} & $\mathrm{CH}$ & $0.044^{*}$ \\
\hline & putamen & $0.047^{\star}$ \\
\hline & parietal WM & $0.04^{*}$ \\
\hline $\mathrm{MB} \geq 1$ and $\mathrm{WMH} \geq 1$ and $\mathrm{PVS} \geq 2$ & $\mathrm{CH}$ & $0.044^{*}$ \\
\hline $\mathrm{MB} \geq 1, \mathrm{WMH} \geq 1$ and $\mathrm{PVS} \geq 3$ & $\mathrm{CH}$ & $0.044^{*}$ \\
\hline \multirow[t]{2}{*}{$\mathrm{WMH} \geq 2$} & putamen & 0.065 \\
\hline & parietal WM & 0.055 \\
\hline $\mathrm{WMH} \geq 2$ and $\mathrm{PVS} \geq 2$ & parietal WM & 0.055 \\
\hline
\end{tabular}

Based on the assumption that CSVD features on MRI represented a more advanced stage of diabetes than no CSVD features on MRI, ${ }^{20}$ diabetes patients were further divided into diabetes with CSVD and diabetes without CSVD. The effect of diabetes on $K_{\text {trans }}$ of $\mathrm{GM}$ after age adjustment was shown in diabetes with CSVD group ( $p=0.035$, permutation test for 10000 random resamples, Fig. $2 \mathrm{H}$ ) but not in diabetes without CSVD group ( $p=0.503$, permutation test for 10000 random resamples, Fig. 2I).

Spatial dynamics: $\mathrm{K}_{\text {trans }}$ of WM was increased in all diabetic patients, whereas $\mathrm{K}_{\text {trans }}$ of $\mathrm{GM}$ was increased in diabetic patients with CSVD 
To examine the spatial dynamics of $K_{\text {trans }}$ in diabetes, the $K_{\text {trans }}$ values of WM and GM were analyzed separately. The $K_{\text {trans }}$ of the WM was increased in all patients with diabetes, irrespective of the absence or presence of CSVD ( $p=0.04,0.02$, respectively, Student's $t$-test, Fig. $3 E)$. The $K_{\text {trans }}$ of the GM was only increased in diabetes with CSVD group that had an advanced diabetes status $(P=0.006$, Student's $t$-test, Fig. $3 F$ ). In addition, among all diabetic patients, the $K_{\text {trans }}$ of the GM was higher in diabetes with CSVD group than in diabetes without CSVD group ( $P=0.023$, Student's $t$-test, Fig. 3F).

Regarding the WM, parietal region showed an increase in $K_{\text {trans }}$ in all patients with diabetes (diabetes without CSVD vs. control, $P=0.04$; diabetes with CSVD vs. control, $P=0.016$; Student's $t$-test, Fig. $3 G$ ), whereas frontal WM, only showed a marginal statistical significance $(P=0.06$, Student's $t$-test, Fig. $3 G)$. Regarding the $\mathrm{GM}$, the $K_{\text {trans }} \mathrm{s}$ of the putamen and $\mathrm{CH}$ were higher in diabetes with CSVD group (diabetes with CSVD vs. control, $P=0.027$; diabetes with CSVD vs. diabetes without CSVD, $P=0.021$, Student's $t$ test, Fig. $3 \mathrm{H}$ ). In summary, in diabetic patients, the increased $K_{\text {trans }}$ of the WM was the most prominent in the parietal $\mathrm{WM}$, and the increased $K_{\text {trans }}$ of the $\mathrm{GM}$ was mainly found in the $\mathrm{CH}$ and putamen.

\section{$\mathrm{K}_{\text {trans }}$ was not correlated with the HbA1c level.}

The HbA1c level, an averaged status of recent glycaemic control, in patients who underwent DCE-MRI was not correlated with the $K_{\text {trans }}$ of the WM or GM $\left(r^{2}=0.0157\right.$ and 0.0176 , respectively, Pearson's correlation). Even analyzing diabetes without CSVD and diabetes with CSVD groups separately, the correlation between the $\mathrm{HbA} 1 \mathrm{c}$ level and $K_{\text {trans }}$ was not observed. We further divided patients with diabetes according to their recent glycaemic control into two groups, $\mathrm{HbA} 1 \mathrm{c}<8$ and $\mathrm{HbA} 1 \mathrm{c} \geq 8$. The $K_{\text {trans }}$ of the WM or GM did not significantly differ between the two groups $(P=0.235$ and 0.173 , respectively, Student's t-test).

\section{High CSVD score correlated with the increased $\mathrm{K}_{\text {trans }}$ in all patients with CSVD.}

We further clarify whether the increase in $K_{\text {trans }}$ generally presents in all CSVDs or is a specific feature for diabetes-related CSVD. Nondiabetic patients with CSVD were enrolled ( $\mathrm{n}=13$ [7 men and six women], aged $74.9 \pm 11$ years). Their sex, age, the prevalence of hypertension or dementia, the stage of CKD, the total CSVD score, the scores of individual CSVD items, and the $K_{\text {trans }}$ values of the five ROls did not differ from the diabetic patients with CSVD (Table 3). Both in diabetes CSVD and nondiabetes CSVD groups, their $K_{\text {trans }}$ of WM, GM, and total $K_{\text {trans }}$ were higher than the control group (Fig. 3I), suggesting the increase in $K_{\text {trans }}$ presents in all CSVDs. 
Table 3

Comparisons of demographic data between patients with CSVD with and without diabetes

\begin{tabular}{|c|c|c|c|}
\hline & Diabetes with CSVD & Non-diabetes CSVD & p-value \\
\hline & $(\mathrm{N}=13)$ & $(\mathrm{N}=12)$ & \\
\hline Gender (Female) $(\mathrm{N}, \%)$ & $4,30.7$ & 9,75 & 0.165 \\
\hline Age $($ mean $\pm S D)$ & $68.78 \pm 10.45$ & $74.9 \pm 11.06$ & 0.94 \\
\hline Hypertension (N,\%) & $8,61.5$ & $7,58.3$ & 0.11 \\
\hline Dementia $(\mathrm{N}, \%)$ & $10,76.9$ & 9,75 & 0.57 \\
\hline CKD stage (median, [IQR]) & $2[1.5,3]$ & $2[1,2]$ & 0.31 \\
\hline Total CSVD score & $3[2.5,3]$ & $3[2,5]$ & 0.301 \\
\hline PVS score & $0[0,1]$ & $1[0,1]$ & 0.393 \\
\hline Lacune score & $1[0,1]$ & $1[0,1]$ & 0.99 \\
\hline WMH score & $1[1,1]$ & $1[1,1]$ & 0.627 \\
\hline MB score & $1[0.5,1]$ & $1[1,2]$ & 0.195 \\
\hline Total $K_{\text {trans }}$ & $0.035 \pm 0.028$ & $0.027 \pm 0.02$ & 0.449 \\
\hline WM $K_{\text {trans }}$ & $0.023 \pm 0.025$ & $0.027 \pm 0.03$ & 0.756 \\
\hline Frontal WM $K_{\text {trans }}$ & $0.013 \pm 0.017$ & $0.0194 \pm 0.0197$ & 0.431 \\
\hline Parietal WM $K_{\text {trans }}$ & $0.033 \pm 0.036$ & $0.0247 \pm 0.0221$ & 0.474 \\
\hline GM $K_{\text {trans }}$ & $0.046 \pm 0.036$ & $0.032 \pm 0.022$ & 0.256 \\
\hline $\mathrm{CH} K_{\text {trans }}$ & $0.032 \pm 0.029$ & $0.023 \pm 0.02$ & 0.365 \\
\hline Putamen $K_{\text {trans }}$ & $0.069 \pm 0.072$ & $0.045 \pm 0.032$ & 0.219 \\
\hline Thalamus $K_{\text {trans }}$ & $0.03 \pm 0.029$ & $0.027 \pm 0.02$ & 0.803 \\
\hline \multicolumn{4}{|c|}{$\begin{array}{l}\text { CKD, chronic kidney disease; SD, standard deviation; CSVD, cerebral small vessel disease; PVS, } \\
\text { perivascular space; WMH, white matter hyperintensity; MB, microbleed; WM, white matter; GM, grey } \\
\text { matter; CH, caudate head. }\end{array}$} \\
\hline
\end{tabular}

The increased $\mathrm{K}_{\text {trans }}$ in diabetes and nondiabetes CSVD correlated with distinct CSVD items

Because the DCE-MRI protocol is time-consuming, it may not be routinely used in conventional medical practice. In addition, DCE-MRI is highly dependent on patients' cooperation and is challenging to perform 
in patients with moderate to severe dementia. To improve the accessibility and applicability of the estimation of BBB permeability, we utilized the individual items of MRI CSVD scores, which are more generally available, to indicate the increase in $K_{\text {trans }}$.

Initially, in recruiting subjects, CSVD was defined as MRI CSVD score $>1$. Any of the 4 CSVD items can contribute a positive score. However, the correlation between an individual item and $K_{\text {trans }}$ was low and varied (Kendall's tau between total $K_{\text {trans }}$ and lacune numbers, total $K_{\text {trans }}$ and PVS numbers, 0.218 and -0.22 , respectively). Thus, we tried to find better combinations of each MRI CSVD item with different thresholds to indicate $K_{\text {trans }}$.

For patients with diabetes, all of them were reclassified according to the various cutoff points of each CSVD item. For instance, patients with diabetes were reclassified into two groups, according to $\mathrm{WMH}<1$ and $\mathrm{WMH} \geq 1$. Subsequently, the $K_{\text {trans }}$ values of the newly generated two groups were compared. For the four CSVD items, $\mathrm{WMH}$ values were assigned four grades $(\mathrm{WMH}=0,1,2$, or 3$)$. The PVS ranged from 0 to $>20$, microbleeds ranged from 0 to $>15$, and lacunes ranged from 0 to 5 . The combinations of the aforementioned items with various cutoff values generated 4500 classification criteria, including the criterion of a single item and the criteria of $\geq 2$ combined items. Among 4500 criteria, any one generated two groups with extremely uneven sample sizes ( $\mathrm{n}<8$ in one group) was discarded. Finally, 76 classification criteria remained. After dividing patients with diabetes using any of the 76 criteria, and comparing the $K_{\text {trans }}$ of the five ROIs between two groups, finally only six criteria generated two groups of statistically different $K_{\text {trans }}$.

Among the four MRI features of CSVD, the presence of microbleeds was the most sensitive indicator for a significantly increased $K_{\text {trans }}$ in multiple brain regions, including the $\mathrm{CH}$, putamen, and parietal WM (Table 2). The addition of other CSVD items to the microbleeds, including PVS $\geq 2$ and $W M H \geq 2$, did not change the statistical result. In patients with no microbleeds on $\mathrm{MRI}, \mathrm{WMH} \geq 2$ alone showed marginal significance in suggesting an increased $K_{\text {trans }}$ in the putamen and parietal WM $(\mathrm{P}=0.065$ and $\mathrm{P}=0.055$, respectively). The presence of the PVS or lacune alone did not indicate an increased $K_{\text {trans }}$ in diabetic patients.

\section{$\mathrm{K}_{\text {trans }}$ correlated with distinct CSVD features in non-diabetic patients.}

To test the hypothesis that diabetes-related CSVD may have distinct pathophysiology from nondiabetes CSVD, the aforementioned methods were also applied to non-diabetic patients. Similar to diabetic patients, the presence of microbleeds suggested an increase in $K_{\text {trans }}$ in non-diabetic patients with CSVD (Table 4). In contrast to diabetic patients, the presence of lacune alone or multiple PVSs $(\geq 6)$ alone indicated an increase in $K_{\text {trans }}$ in non-diabetic patients with CSVD. Although Moderate WMH $(\geq 2)$ showed marginal significance in indicating $K_{\text {trans }}$ in the diabetes group, it was not an indicator in the nondiabetes group. 
Table 4

Statistical power of various combinations of CSVD items in determining the $K_{\text {trans }}$ in nondiabetes group

\begin{tabular}{|c|c|c|}
\hline Criteria & Sensitive region & p-value \\
\hline \multirow[t]{2}{*}{$M B \geq 1$} & $\mathrm{CH}$ & $0.016^{*}$ \\
\hline & frontal WM & $0.013^{*}$ \\
\hline \multirow[t]{2}{*}{$M B \geq 2$} & $\mathrm{CH}$ & $0.0086^{\star \star}$ \\
\hline & frontal WM & $0.01 *$ \\
\hline \multirow[t]{2}{*}{$M B \geq 1$ and $P V S \geq 4$} & $\mathrm{CH}$ & $0.003^{* \star}$ \\
\hline & frontal WM & $0.02^{\star}$ \\
\hline$M B \geq 2$ and $P V S \geq 4$ & $\mathrm{CH}$ & $0.0017 * \star$ \\
\hline$M B \geq 2$ and $P V S \geq 7$ & frontal WM & $0.027^{*}$ \\
\hline \multirow[t]{2}{*}{$\mathrm{MB} \geq 2$ and $\mathrm{WMH} \geq 2$} & $\mathrm{CH}$ & 0.05 \\
\hline & frontal WM & 0.075 \\
\hline Lacune $\geq 1$ & $\mathrm{CH}$ & $0.032^{\star}$ \\
\hline \multirow[t]{2}{*}{$P V S \geq 6$} & $\mathrm{CH}$ & 0.077 \\
\hline & frontal WM & $0.029 *$ \\
\hline \multirow[t]{2}{*}{$P V S \geq 6$ and $\mathrm{WMH} \geq 1$} & $\mathrm{CH}$ & $0.047^{*}$ \\
\hline & frontal WM & $0.024^{*}$ \\
\hline$P V S \geq 7$ & frontal WM & $0.035^{\star}$ \\
\hline$P V S \geq 8$ & frontal WM & $0.028^{*}$ \\
\hline \multicolumn{3}{|c|}{$\begin{array}{l}\text { CKD, chronic kidney disease; SD, standard deviation; CSVD, cerebral small vessel disease; PVS, } \\
\text { perivascular space; WMH, white matter hyperintensity; MB, microbleed; WM, white matter; GM, grey } \\
\text { matter; } \mathrm{CH} \text {, caudate head; F, frontal white matter }\end{array}$} \\
\hline
\end{tabular}

\section{Discussion}

This study demonstrated the early presence of cerebral microangiopathy in diabetic patients with distinct mechanisms from other CSVD, supported by the following evidence: First, the increased $K_{\text {trans }}$ in WM was prior to the presence of visible CSVD features on MRI. Second, the increased $K_{\text {trans }}$ in GM developed in advanced diabetes. Third, the increased $K_{\text {trans }}$ was associated with the progress of diabetes, not with the recent glycemic control. Forth, among the four CSVD features on MRI, the presence of microbleeds indicated the increased $K_{\text {trans }}$ in all CSVDs. Fifth, the presence of lacunae or PVS alone was not a good indicator for the increased $K_{\text {trans }}$ in diabetes-related CSVD but can be suitable for non-diabetic CSVDs. 
The differential correlations between $K_{\text {trans }}$ and each CSVD items of MRI suggested the distinct pathophysiology between diabetes-related CSVD and other CSVDs and supported the high heterogeneity in all CSVDs.

Diabetes-related CSVD is a manifestation of microangiopathy involving the brain ${ }^{4}$. Our results supported the notion that the brain might be involved concurrently with other target organs, as early as the retina and kidneys ${ }^{4,21-23}$. A microvascular leakage in the brain might be similar to the extravasation of the retinal vessels. These target organ damages may share similar mechanisms, by which reactive oxygen species, dysregulation of vascular endothelial growth factor, and other growth factors lead to endothelial dysfunction and subsequently result in damages of filtration barrier and sclerosis ${ }^{24-26}$. Increased BBB permeability enables toxic molecules to enter the brain parenchyma, thus initiating multiple pathways of neurodegeneration ${ }^{27}$. Subsequently, cognitive decline can develop. In our subjects, a higher prevalence of dementia was found in diabetes with the CSVD group than in the non-CSVD group, supporting the epidemiological observation of the increased risk of cognitive decline in the progression of diabetes ${ }^{6,28}$.

Although all CSVDs shared similar features on conventional MRI, DCE-MRI enabled us to visualize early differences in functions between various subtypes of CSVDs. However, the DCE-MRI protocol was timeconsuming and highly dependent on patient cooperation. Any motion artifact compromised the accuracy of measurements. Sedative drugs were not used, considering the uncertainty of their effects on BBB permeability. Therefore, patients with moderate to severe dementia were not examined. In addition, patients with advanced CKD, which represented a more advanced diabetes status, were excluded from the DCE-MRI examination considering the risk of gadolinium nephrotoxicity. These limited the generality of the results.

In brain images with too many lacunae or PVSs, the estimation of the parenchymal intensity was difficult because no homogeneous ROI without any fluid space could be observed. Thus, these patients were excluded from our analysis. To overcome the limitations above and broaden the applicability of cerebral permeability using current commonly available methods for patients who cannot undergo DCE-MRI, CSVD items of conventional MRI may help physicians assess the BBB integrity.

\section{Conclusion}

This study demonstrated that the increase of cerebral permeability occurred before the image features of CSVD could be visualized on conventional MRI. The increased permeability was associated with longlasting disease course rather than recent glycaemic control. When DCE-MRI is not available, the commonly applicable CSVD features on conventional MRI, such as microbleeds and moderate WMH, can indicate the increased permeability. Cerebral vascular permeability in diabetic and non-diabetic patients with CSVD correlated with different CSVD items, suggesting distinct pathogenesis for diabetic cerebral microangiopathy.

\section{Abbreviations}


CSVD = Cerebral small vessel disease; $\mathrm{BBB}=$ blood-brain barrier; $\mathrm{DCE}-\mathrm{MRI}=$ dynamic contrast-enhanced $\mathrm{MRI} ; \mathrm{IRB}=$ Institutional Review Board; $\mathrm{CKD}$ = chronic kidney disease; T1WI = T1-weighted images; FLAIR = fluid-attenuated inversion recovery; T1-FFE = T1-weighted fast-field echo; Gd-DOTA = gadoterate meglumine; $\mathrm{WM}=$ white matter; $\mathrm{GM}=$ grey matter; $\mathrm{ROI}$ = regions of interest; $\mathrm{CH}$ = caudate head; PVS = perivascular space; $\mathrm{WMH}=$ white matter hyperintensity;

\section{Declarations}

\section{Acknowledgments}

We thank Dr. Shih-Kai Chu in the Clinical Medicine Research Center, National Cheng Kung University Hospital, for statistics support.

\section{Contributor}

Design and conceptualization of the study: YTS and YCC. Acquisition and analysis of the data: YCC and BZL. Recruitment of the subjects: YTS. Interpretation of the data: YCC and YTS. Drafting and revising the manuscript: YCC, BZL, YTS. Visual analysis of the MR images: YCC and YTS. Processing the MR images, analysis of the data: YCC, BZL, YCS.

\section{Funding}

The Ministry of Science and Technology, Taiwan (MOST 109-2635-B-006-003, MOST 109 -2115-M-006 -018 -MY2) and National Cheng Kung University Hospital (NCKUH-11004035) supported this study.

\section{Competing interests}

The authors report no competing interests.

\section{Ethics approval}

The Institutional Review Board of National Cheng Kung University Hospital approved the study protocol (A-BR-106-081).

\section{Consent for publication}

Not applicable.

\section{Data sharing}

Data are available upon reasonable request. Yuan-Ting Sun ORCID 0000-0003-2899-1136

\section{References}


1. Ihara M, Yamamoto Y. Emerging Evidence for Pathogenesis of Sporadic Cerebral Small Vessel Disease. Stroke. Feb 2016;47(2):554-60. doi:10.1161/STROKEAHA.115.009627

2. Manschot SM, Brands AM, van der Grond J, et al. Brain magnetic resonance imaging correlates of impaired cognition in patients with type 2 diabetes. Diabetes. Apr 2006;55(4):1106-13. doi:10.2337/diabetes.55.04.06.db05-1323

3. Funnell C, Doyle-Waters MM, Yip S, Field T. What is the relationship between type 2 diabetes mellitus status and the neuroradiological correlates of cerebral small vessel disease in adults? Protocol for a systematic review. Syst Rev. Jan 17 2017;6(1):7. doi:10.1186/s13643-017-0410-1

4. Umemura T, Kawamura T, Hotta N. Pathogenesis and neuroimaging of cerebral large and small vessel disease in type 2 diabetes: A possible link between cerebral and retinal microvascular abnormalities. Journal of diabetes investigation. Mar 2017;8(2):134-148. doi:10.1111/jdi.12545

5. Chen R, Ovbiagele B, Feng W. Diabetes and Stroke: Epidemiology, Pathophysiology, Pharmaceuticals and Outcomes. The American journal of the medical sciences. Apr 2016;351(4):380-6. doi:10.1016/j.amjms.2016.01.011

6. Biessels GJ, Despa F. Cognitive decline and dementia in diabetes mellitus: mechanisms and clinical implications. Nat Rev Endocrinol. Oct 2018;14(10):591-604. doi:10.1038/s41574-018-0048-7

7. Guariguata L, Whiting DR, Hambleton I, Beagley J, Linnenkamp U, Shaw JE. Global estimates of diabetes prevalence for 2013 and projections for 2035. Diabetes research and clinical practice. Feb 2014;103(2):137-49. doi:10.1016/j.diabres.2013.11.002

8. Raja R, Rosenberg GA, Caprihan A. MRI measurements of Blood-Brain Barrier function in dementia: A review of recent studies. Neuropharmacology. May 15 2018;134(Pt B):259-271. doi:10.1016/j.neuropharm.2017.10.034

9. Sanahuja J, Alonso N, Diez J, et al. Increased Burden of Cerebral Small Vessel Disease in Patients With Type 2 Diabetes and Retinopathy. Diabetes Care. Sep 2016;39(9):1614-20. doi:10.2337/dc152671

10. Starr JM, Wardlaw J, Ferguson K, MacLullich A, Deary IJ, Marshall I. Increased blood-brain barrier permeability in type II diabetes demonstrated by gadolinium magnetic resonance imaging. Journal of neurology, neurosurgery, and psychiatry. Jan 2003;74(1):70-6. doi:10.1136/jnnp.74.1.70

11. Bergamino M, Bonzano L, Levrero F, Mancardi GL, Roccatagliata L. A review of technical aspects of T1-weighted dynamic contrast-enhanced magnetic resonance imaging (DCE-MRI) in human brain tumors. Physica medica : PM : an international journal devoted to the applications of physics to medicine and biology : official journal of the Italian Association of Biomedical Physics. Sep 2014;30(6):635-43. doi:10.1016/j.ejmp.2014.04.005

12. American Diabetes A. 2. Classification and Diagnosis of Diabetes: Standards of Medical Care in Diabetes-2020. Diabetes Care. Jan 2020;43(Suppl 1):S14-S31. doi:10.2337/dc20-S002

13. American Diabetes A. 2. Classification and Diagnosis of Diabetes: Standards of Medical Care in Diabetes-2019. Diabetes Care. Jan 2019;42(Suppl 1):S13-S28. doi:10.2337/dc19-S002 
14. American Diabetes A. 2. Classification and Diagnosis of Diabetes: Standards of Medical Care in Diabetes-2018. Diabetes Care. Jan 2018;41(Suppl 1):S13-S27. doi:10.2337/dc18-S002

15. Levey AS, Coresh J, Balk E, et al. National Kidney Foundation practice guidelines for chronic kidney disease: evaluation, classification, and stratification. Annals of internal medicine. Jul 15 2003;139(2):137-47. doi:10.7326/0003-4819-139-2-200307150-00013

16. Tomson CRV, Cheung AK, Mann JFE, et al. Management of Blood Pressure in Patients With Chronic Kidney Disease Not Receiving Dialysis: Synopsis of the 2021 KDIGO Clinical Practice Guideline. Annals of internal medicine. Jun 22 2021;doi:10.7326/M21-0834

17. Staals J, Makin SD, Doubal FN, Dennis MS, Wardlaw JM. Stroke subtype, vascular risk factors, and total MRI brain small-vessel disease burden. Neurology. Sep 30 2014;83(14):1228-34. doi:10.1212/WNL.0000000000000837

18. Heye AK, Thrippleton MJ, Armitage PA, et al. Tracer kinetic modelling for DCE-MRI quantification of subtle blood-brain barrier permeability. Neuroimage. Jan 15 2016;125:446-455. doi:10.1016/j.neuroimage.2015.10.018

19. van de Haar HJ, Burgmans S, Jansen JF, et al. Blood-Brain Barrier Leakage in Patients with Early Alzheimer Disease. Radiology. Nov 2016;281(2):527-535. doi:10.1148/radiol.2016152244

20. Del Bene A, Ciolli L, Borgheresi L, Poggesi A, Inzitari D, Pantoni L. Is type 2 diabetes related to leukoaraiosis? an updated review. Acta Neurol Scand. Sep 2015;132(3):147-55. doi:10.1111/ane.12398

21. Lizarraga KJ, Chunga N, Yannuzzi NA, Flynn HW, Jr., Singer C, Lang AE. The retina as a window to the basal ganglia: Systematic review of the potential link between retinopathy and hyperkinetic disorders in diabetes. Parkinsonism Relat Disord. Nov 2020;80:194-198. doi:10.1016/j.parkreldis.2020.10.025

22. Umemura T, Kawamura T. Retinopathy: A sign of cerebral small vessel disease in diabetes? $J$ Diabetes Investig. Jul 2017;8(4):428-430. doi:10.1111/jdi.12602

23. Li J, Pan J, Li B, et al. Positive correlation between cognitive impairment and renal microangiopathy in patients with type 2 diabetic nephropathy: a multicenter retrospective study. J Int Med Res. Dec 2018;46(12):5040-5051. doi:10.1177/0300060518789299

24. Haj Najeeb B, Simader C, Deak G, et al. The Distribution of Leakage on Fluorescein Angiography in Diabetic Macular Edema: A New Approach to Its Etiology. Investigative ophthalmology \& visual science. Aug 1 2017;58(10):3986-3990. doi:10.1167/iovs.17-21510

25. Satchell SC, Tooke JE. What is the mechanism of microalbuminuria in diabetes: a role for the glomerular endothelium? Diabetologia. May 2008;51(5):714-25. doi:10.1007/s00125-008-0961-8

26. Campos A, Campos EJ, Martins J, Ambrosio AF, Silva R. Viewing the choroid: where we stand, challenges and contradictions in diabetic retinopathy and diabetic macular oedema. Acta ophthalmologica. Aug 2017;95(5):446-459. doi:10.1111/aos.13210

27. Sweeney MD, Sagare AP, Zlokovic BV. Blood-brain barrier breakdown in Alzheimer disease and other neurodegenerative disorders. Nat Rev Neurol. Mar 2018;14(3):133-150.

doi:10.1038/nrneurol.2017.188

Page $16 / 20$ 
28. Biessels GJ, Staekenborg S, Brunner E, Brayne C, Scheltens P. Risk of dementia in diabetes mellitus: a systematic review. Lancet Neurol. Jan 2006;5(1):64-74. doi:10.1016/S1474-4422(05)70284-2

\section{Figures}

A

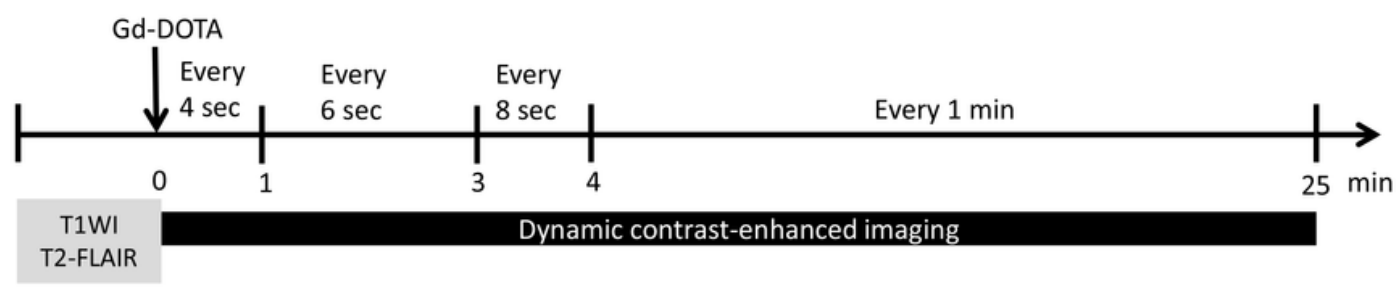

Fig 1

B
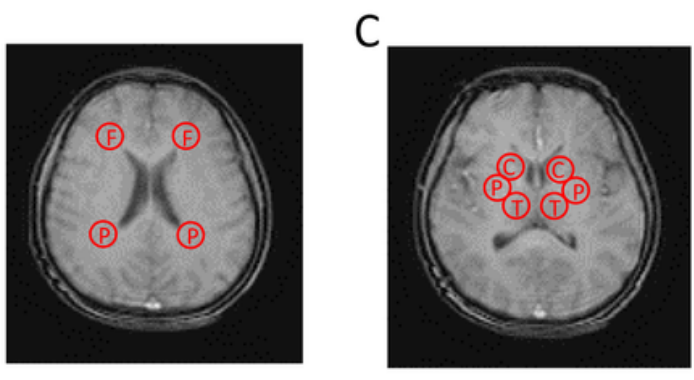

$\mathrm{F}$

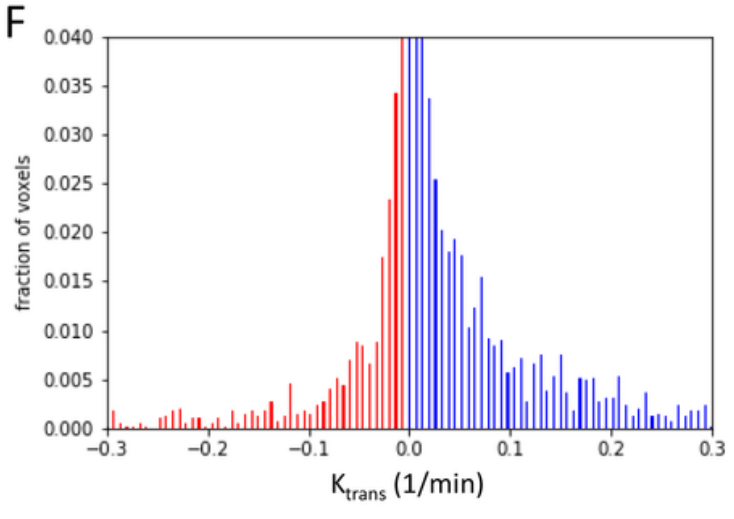

$\mathrm{D}$
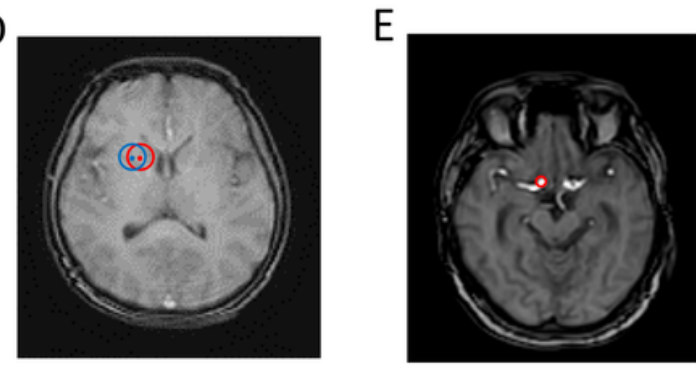

G

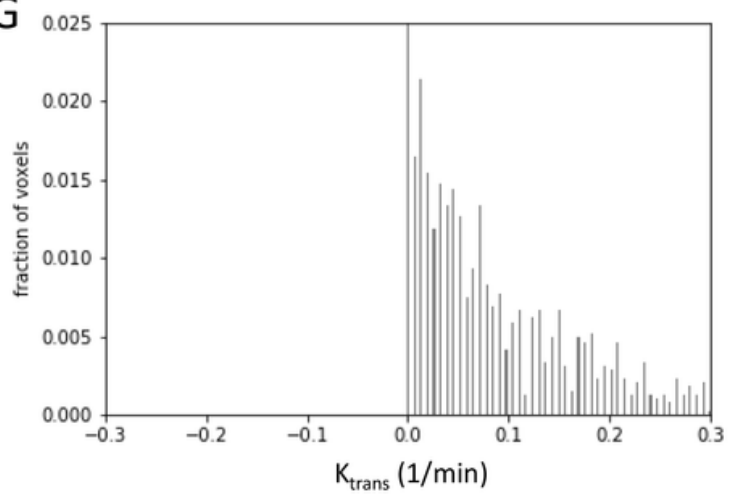

\section{Figure 1}

Image processing. (A) Imaging protocols included structural images (T1WI and T2-FLAIR images) and dynamic imaging. The time bar shows dynamic imaging intervals during the 25 -min acquisition time. (BC) Examples showing the two regions of interest (ROIs) in the white matter (WM) (B), namely the frontal and parietal WM, and three ROIs in the grey matter (C), namely the caudate head, putamen, and thalamus.

(D) An example showing the perturbation of ROls. The center and radius of the initial ROI (in red) randomly oscillated within three pixels to obtain another ROI (in blue) that was near to the initially examined ROI (in red). (E) The internal carotid artery was selected and set as the region of plasma in Patlak analysis. (F-G) The Ktrans of each pixel was plotted as a histogram. Red bars indicated negative Ktrans values and were considered as noise (F). The noise was considered to exist with the same distribution on the positive side. By subtracting noise (red bars) from the original histogram (blue bars), tissue permeability was observed (black bars in G). X-axis, Ktrans; Y-axis, the fraction of voxels. F, frontal; 
P, parietal; C, caudate head; P, putamen; T, thalamus; Gd-DOTA, gadoterate meglumine; T1WI, T1-weighted image; T2-FLAIR, T2-weighted fluid-attenuated inversion recovery.
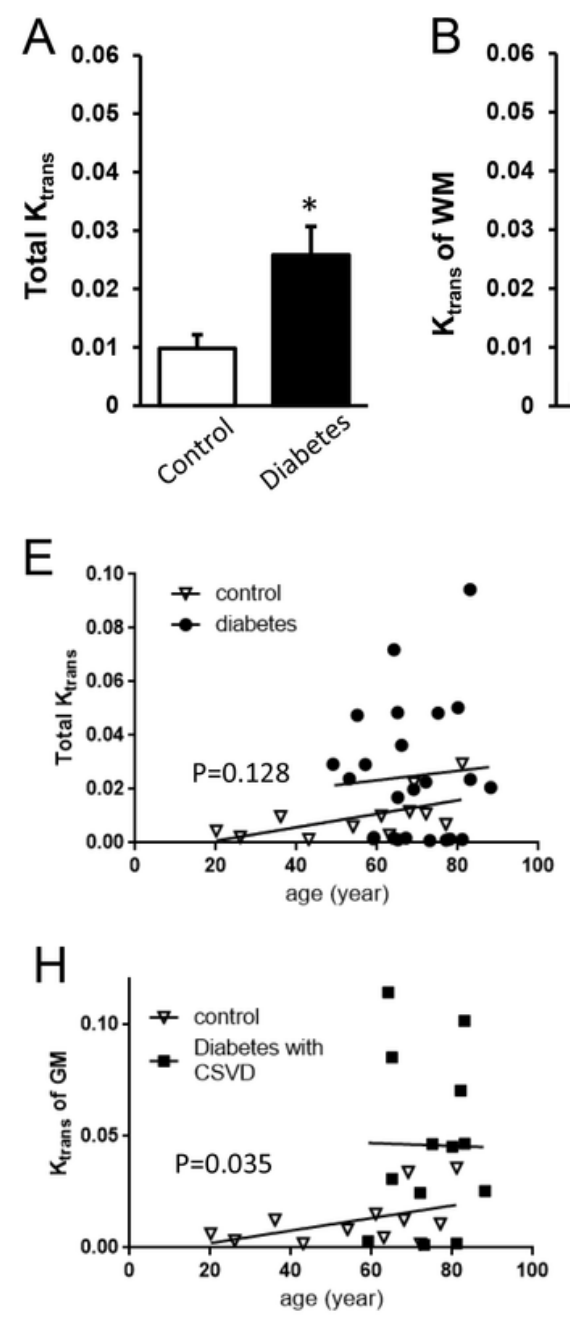
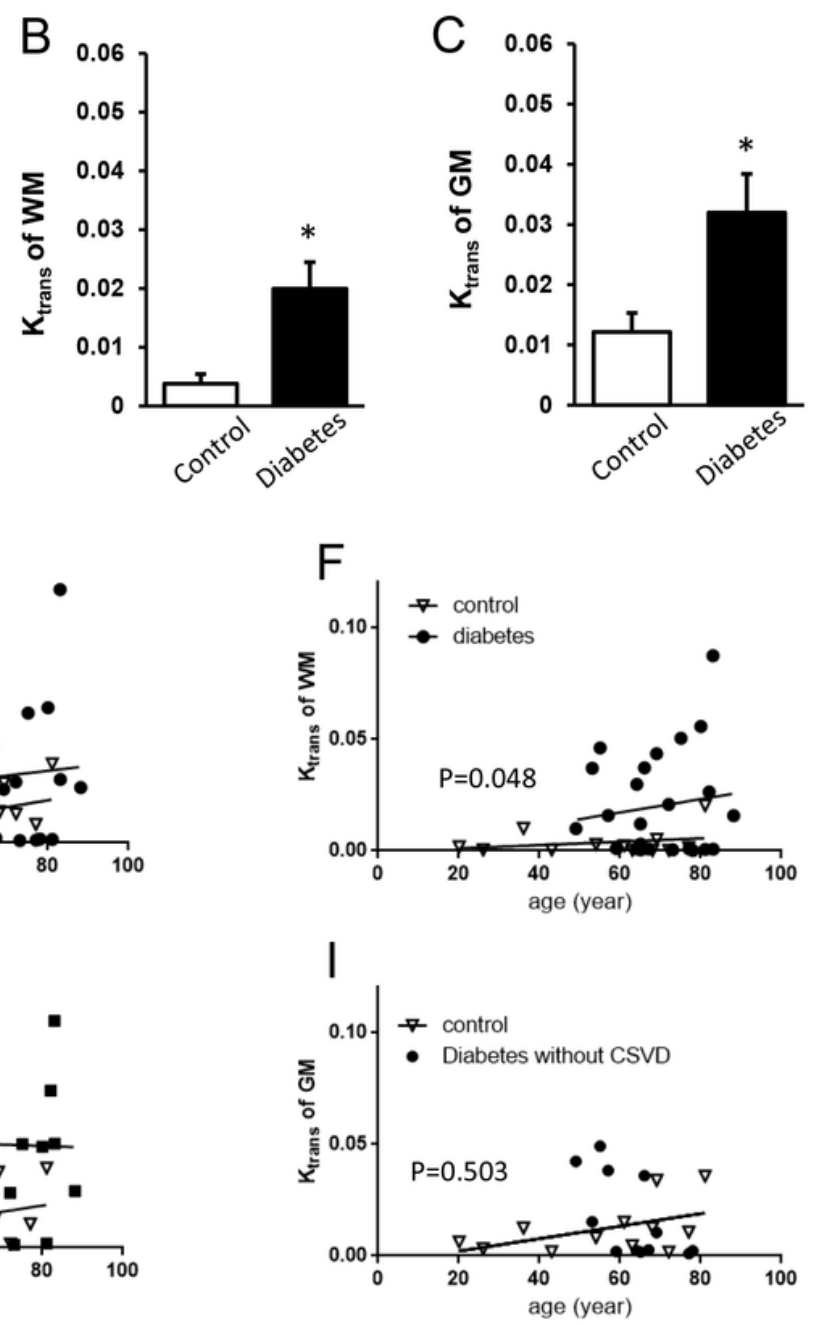
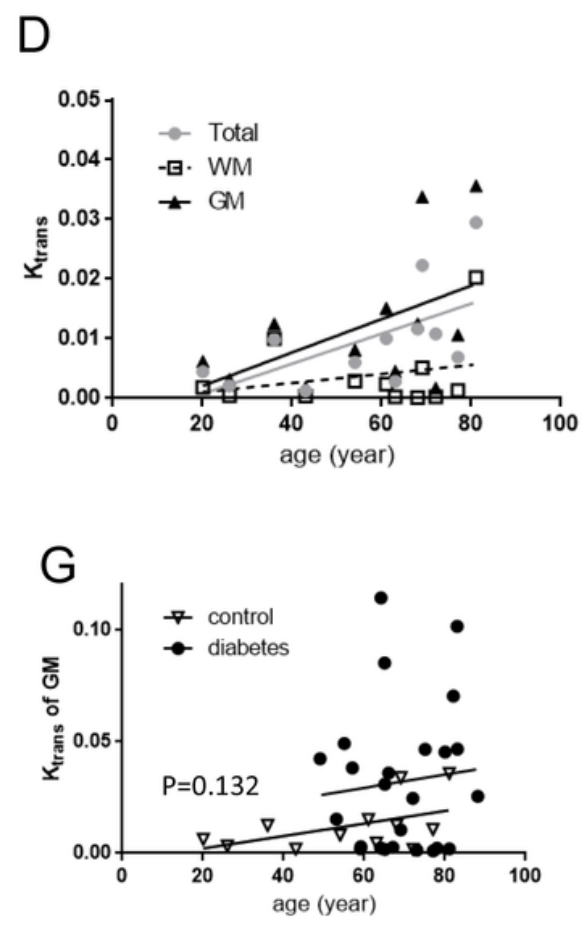

\section{Figure 2}

Comparisons of Ktrans among control, diabetes, and diabetes with CSVD groups. (A-C) Comparisons of total Ktrans (A), Ktrans of the white matter (WM) (B), and Ktrans of the grey matter (GM) (C) between patients with diabetes and controls. Unpaired t-test. ${ }^{*}, P<0.05$. (D) The averaged total Ktrans, Ktrans of the WM, and GM plotted against age in the control group. (E-G) The total Ktrans (E), Ktrans of the WM (F), and GM (G) plotted against age in diabetes and control groups. (H-I) The Ktrans of the GM plotted against age in diabetes with CSVD and control group $(\mathrm{H})$ and in diabetes without CSVD and control group (I). X axis, age; $Y$ axis, Ktrans. WM, white matter; GM, grey matter. 


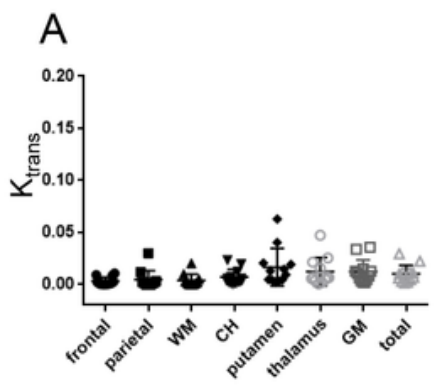

B

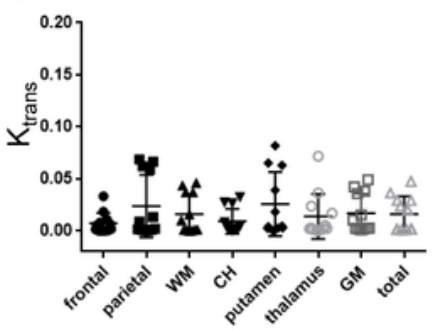

$\mathrm{E}$
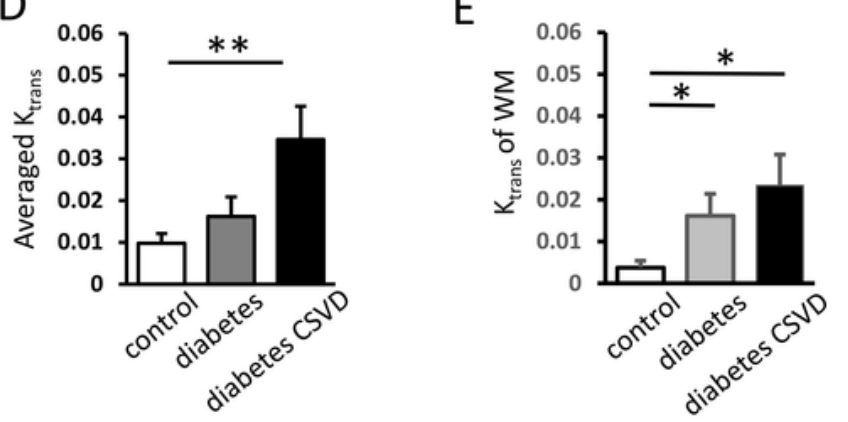

C

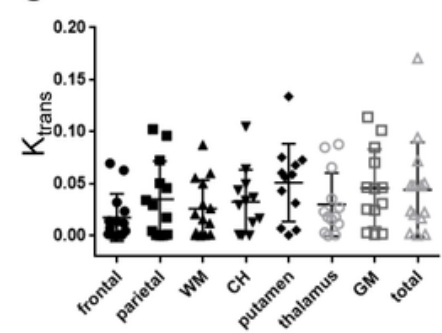

$\mathrm{F}$

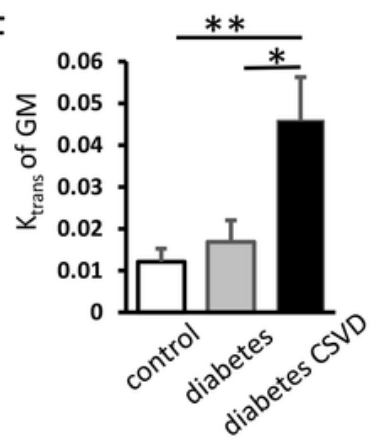

G

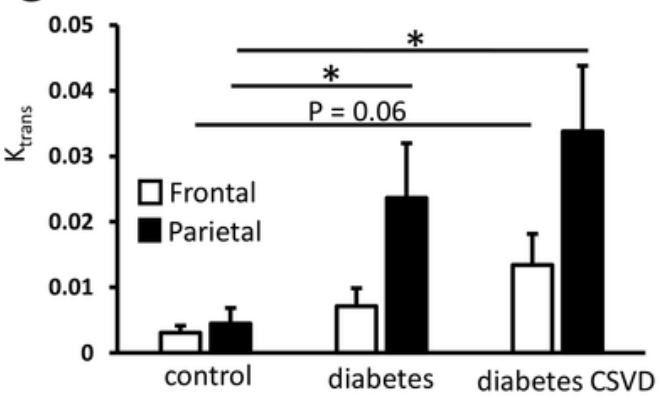

Fig.3

$\mathrm{H}$
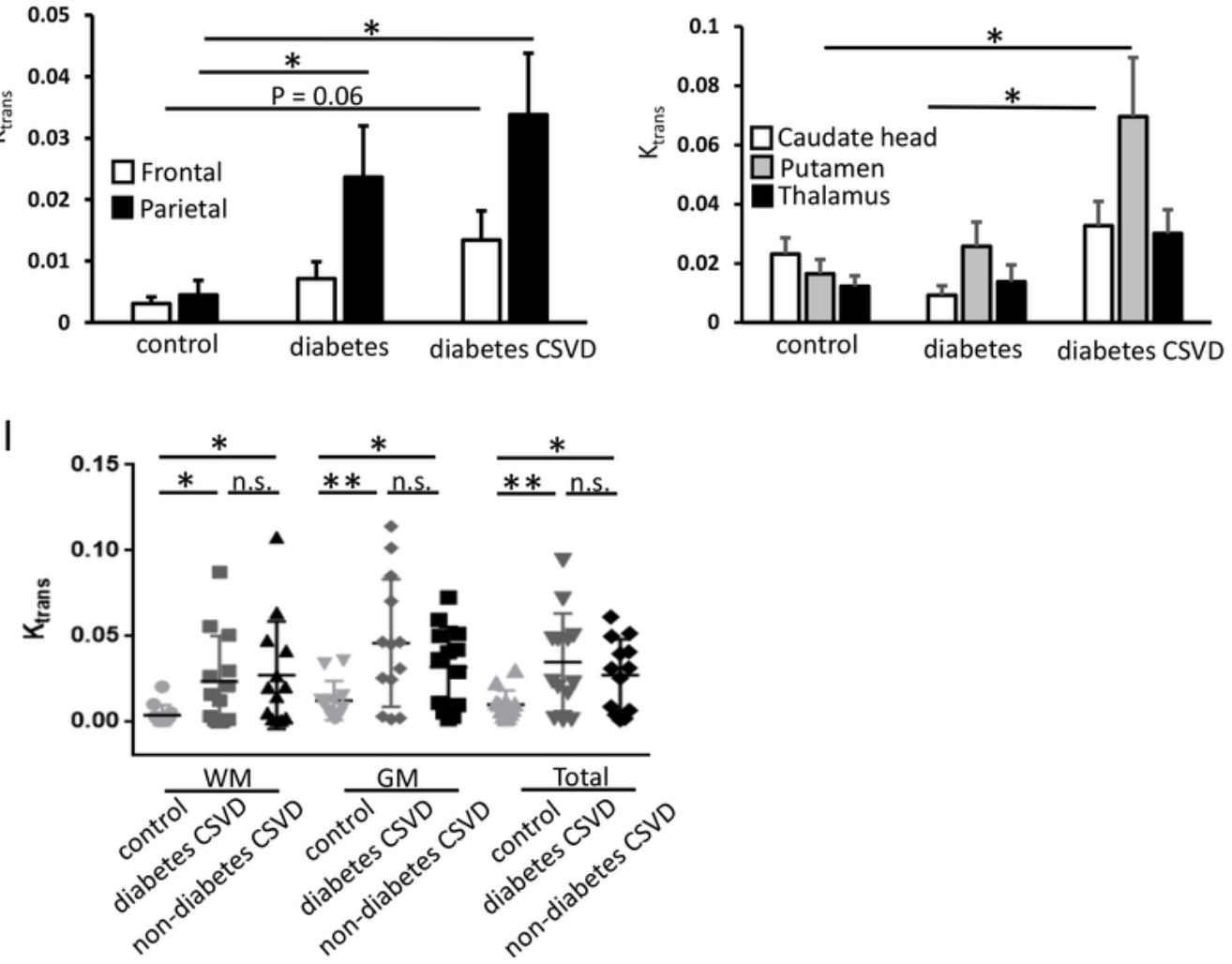

Figure 3

Ktrans of each cerebral area in the control, diabetes, and diabetes with CSVD groups. (A-C) Ktrans of each area in the control (A), diabetes without CSVD (B), and diabetes with CSVD groups (C). (D-F) Comparisons of the averaged total Ktrans (D), Ktrans of the WM (E), and Ktrans of the GM (F) among control, diabetes without CSVD, and diabetes with CSVD groups. (G-H) Comparisons of Ktrans in the frontal and parietal WM $(\mathrm{G})$ and Ktrans in the caudate head $(\mathrm{CH})$, putamen, and thalamus $(\mathrm{H})$ between control, diabetes 
without CSVD, and diabetes with CSVD groups. (I) Comparisons of Ktrans in the WM, GM, and total Ktrans between the control group, CSVD patients with diabetes and CSVD patients without diabetes. Unpaired t-test. NS, no significance; ${ }^{*}, \mathrm{P}<0.05 ; * \star, \mathrm{P}<0.01$. WM, white matter; $\mathrm{GM}$, grey matter; $\mathrm{CH}$, caudate head. 